\title{
A58 METHOTHREXATE DIRECTLY INHIBITS RANKL EXPRESSION AND OSTEOCLAST FORMATION IN VERY EARLY ARTHRITIS
}

S Revu, P Neregård, E af Klint, A I Catrina Rheumatology Unit, Department of Medicine, Karolinska Institute, Karolinska University Hospital, Solna, Sweden

\subsection{6/ard.2010.129593t}

Objective Methotrexate (MTX) is one of the most widely used therapies in rheumatoid arthritis (RA) due to its antiinflammatory and potential bone protection effect. Bone biology is governed by the RANKL/RANK/OPG system that determines the balance between bone formation by osteoblasts and bone resorption by osteoclasts. We investigated the effects of MTX on the RANKL/RANK/OPG system in vivo and in vitro.

Methods 16 patients with newly diagnosed RA (mean disease duration 1 week) were started on MTX $10 \mathrm{mg}$ once a week and increased by $10 \mathrm{mg}$ each week until a stable dose of $20 \mathrm{mg}$ once a week was reached. Patients were naïve for other disease-modifying antirheumatic drugs and allowed to take prednisolone to a maximum dose of $10 \mathrm{mg}$ daily and non-steroidal inflammatory drugs as clinically indicated. Synovial biopsies were obtained by needle arthroscopy at baseline and 8 weeks after initiation of therapy. $x$-rays of hands and feet were obtained at baseline and 1 year after diagnosis. Immunohistochemical analysis was performed to detect RANKL, RANK and OPG in the synovial biopsies. We further investigated the in vitro effect of MTX on synovial fluid-derived mononuclear cells, synovial fluid mononuclear cells (SFMC) (by immunohistochemistry), osteoblasts (by real-time PCR and western blot analysis) and osteoclast formation (tartrate-resistant acid phosphatase (TRAP) staining). Statistical analysis was performed using the Wilcoxon and Mann-Whitney test when appropriate.

Results Nine patients (56\%) were responders to therapy according to EULAR criteria. Two patients had erosions at inclusion in the study and five more developed erosions at 1-year follow-up in both the responder and non-responder groups. MTX treatment decreased synovial inflammation with a significant reduction of synovial cellularity. In parallel, MTX decreased synovial RANK expression and the RANKL/OPG ratio, mainly in the subgroup of RA patients with no radiological progression at 1-year follow-up. We confirmed the effect on RANK expression in SFMC cultured in vitro with MTX. A decrease in the RANKL/OPG ratio was also observed in cultured osteoblasts at both the mRNA and protein levels. MTX blocks osteoclastogenesis from PBMC despite the presence of macrophage colony stimulating factor and RANKL, indicating that MTX directly inhibits osteoclastogenesis.

Conclusions MTX directly affects the RANKL/RANK/OPG system and inhibits osteoclasts formation providing an attractive explanation for the bone-sparing effect of MTX. 